\title{
Modeling PA linearity and efficiency in MIMO transmitters
}

\author{
Filipe M. Barradas, Telmo R. Cunha, Pedro M. Cabral, and José C. Pedro \\ DETI, Instituto de Telecomunicações, Universidade de Aveiro, 3810-193 Aveiro, Portugal
}

\begin{abstract}
Modern transmitter architectures rely on multi input multi output (MIMO) techniques, using several radio-frequency (RF) power amplifiers (PAs) to excite an antenna array. The array elements are often coupled, creating apparent variable loads at the output of each PA. In this system, the behavior of each PA cannot be fully described solely as a function of its input, as it will change according to the coupled signal. Moreover, the impact of the RF mutual coupling is felt not only on the RF output, but also on the direct current (DC) consumption, and thus efficiency, of the device. In this paper, we propose a novel PA modeling approach capable of predicting both the RF output and the absorbed DC current, dependent on the excitation and coupled signals. The proposed model is, therefore, suitable for system level simulations including efficiency and linearity predictions.
\end{abstract}

Index Terms-5G mobile communication, power amplifiers, behavioral modelling, MIMO transmitters.

\section{INTRODUCTION}

MIMO transmitters have been broadly used for RF communications. These transmitters make use of an antenna array, with independently excited elements, to improve the characteristics of the overall system. Antenna arrays typically do not offer perfect isolation between the elements, often presenting some mutual coupling. This becomes especially important with the advent of $5^{\text {th }}$ generation $(5 \mathrm{G})$ communications, which are expected to adopt a massive MIMO configuration $[1,2]$.

If the RF paths are non-isolated, the PAs, responsible for delivering power to the antenna array, cannot be assumed to work under a constant load. In fact, each PA will be subject to an incident wave at the output, which is dependent on the signals generated by each of the other PAs, the input match of its own antenna element, and the coupling between the antenna elements; as seen in Fig. 1 and as described in (1), in terms of the array's $\mathrm{S}$ parameters.

$$
a_{2, i}=\sum_{k=1}^{K} S_{i, k} b_{2, k}
$$

In (1), the PA is connected to the ith element of an array with $K$ elements, and $a_{2, k} / b_{2, k}$ are the incident/reflected waves at the $k^{\prime}$ th PA element (see Fig. 1). In continuous wave (CW) operation, equation (1) shows that the output load of the PA is dependent on the output of each PA whenever nearby PA signals are highly correlated as happens in beam-steering, phased arrays.

Unfortunately, this output load variation will greatly impact the output power, linearity, and efficiency of each PA. This interaction between the PAs and output loads make this system difficult to evaluate. In these cases, simulation is a powerful tool that can be used to study the system's performance. Optimally, the system is simulated with signals close to the

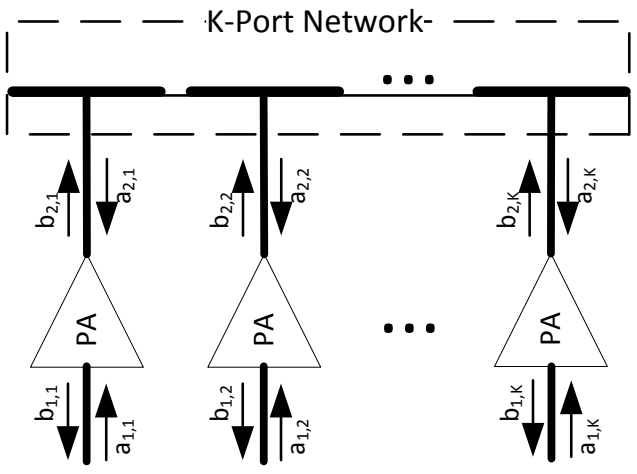

Fig. 1: Antenna array schematic with several RF PAs.

real excitation signals, to obtain accurate predictions. However, using circuit simulation leads to a highly complex model, difficult to simulate in an envelope transient analysis. To solve this problem, nonlinear behavioral models in the envelope domain that take into account the input and output excitations can be developed and used in these simulations.

Previously, the RF behavior of the PA has been investigated when it is excited at both the input and output, and several models have been proposed to accommodate the observed changes under these conditions [3, 4]. However, these models cannot predict the efficiency behavior of the PAs as they do not account for the DC current consumption, which is also sensitive to the output load, or output incident wave.

Power efficiency has become one of the key figures of merit of RF transmitters. It is therefore important to have models that can predict the efficiency behavior under MIMO conditions. In this paper, we propose a measurement and modeling technique that is capable of both predicting linearity and efficiency of a PA working with a non-constant load. This model can then be used for system level simulation of a MIMO transmitter without relying on an underlying circuit simulation of each PA.

\section{CHARACTERIZATION SETUP}

To characterize the behavior of the PAs, the setup shown in Fig. 2 was built. This setup allows simultaneous sampling of the input and output waves of the PA, as well as direct control of the incident waves at the PA ports. Furthermore, the setup also measures the DC current through time, using a current probe, which will allow the modeling of the DC power consumption. Evidently, the setup is designed to allow excitation and measurement of modulated carriers.

As seen in Fig. 2, the setup uses a Rohde \& Schwarz 


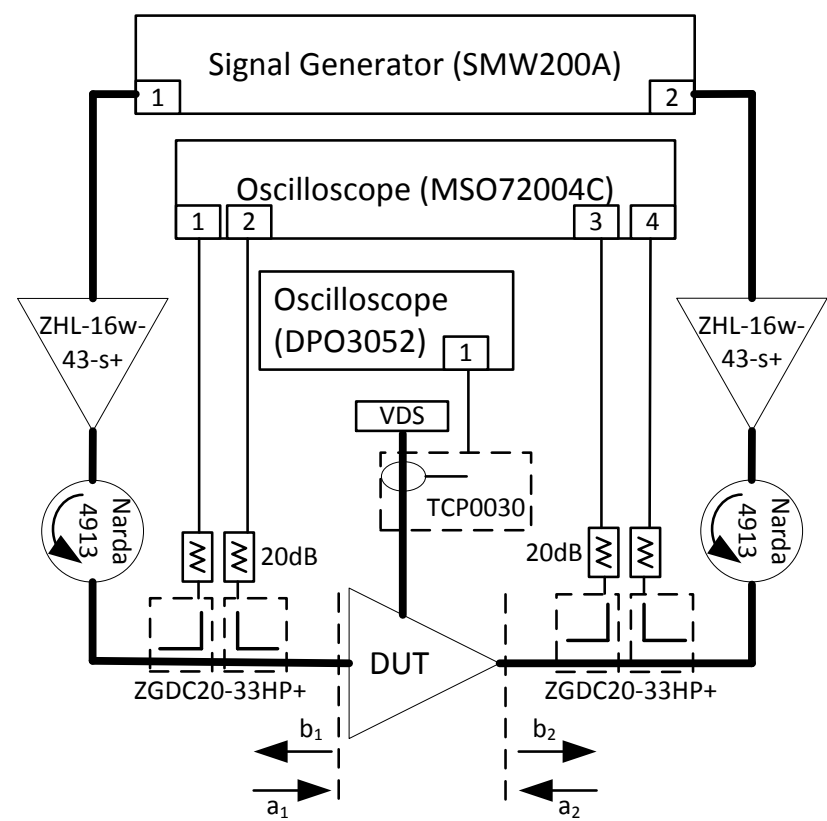

Fig. 2: Characterization setup: the setup allows full control over the excitation waves, as well as measurement of the waves at the PA ports and the DC current.

SMW200A dual channel signal generator, to generate the input and output excitation waves. The signals are amplified using two ZHL-16W-43-S+ drivers from Mini-Circuits and are then fed to the device under test (DUT). Two Narda 4913 isolators are used to guarantee isolation between the drivers and DUT. The waves traveling to and from the DUT are coupled to a Tektronix MSO72004C high speed oscilloscope using four Mini Circuits ZGDC20-33HP+ couplers. The DC drain current is measured using a Tektronix TCP0030 current probe connected to a Tektronix DPO3052 oscilloscope.

Before measuring the DUT, the setup is calibrated using a Short-Open-Load-Through (SOLT) technique to the DUT reference planes. To this relative calibration, a power calibration is added to ensure correct power measurements at the DUT ports. After this calibration has been performed, the channel from the signal generators to the DUT input and output ports is extracted and the RF phase difference between the signal generator channels is calibrated. These steps allow both correct measurement of the waves at the DUT ports and correct generation of the excitation waves at the generators.

\section{MODELING APPROACH}

According to the setup shown in Fig. 2, the controllable inputs are the incident waves at the DUT ports while the outputs are the reflected waves, $b_{1}$ and $b_{2}$, and the absorbed DC current. The main interest is modelling the output wave from the DUT, $b_{2}$, and the absorbed current, which impact system linearity, efficiency and output power. From this perspective $b_{1}$ can be disregarded.

\section{A. RF Behavior}

To describe the RF behavior, we adopted a model from the literature [4], shown in (2).

$$
\begin{gathered}
y_{1}(n)=\sum_{m=0}^{M} f_{m}^{1}\left(\left|a_{1}(n-m)\right|\right) a_{1}(n-m) \\
y_{2}(n)=\sum_{m=0}^{M} f_{m}^{2}\left(\left|a_{1}(n-m)\right|\right) a_{2}(n-m) \\
y_{3}(n)=\sum_{m=0}^{M} f_{m}^{3}\left(\left|a_{1}(n-m)\right|\right) \frac{\left(a_{1}(n-m)\right)^{2}}{\left|a_{1}(n-m)\right|^{2}} a_{2}^{*}(n-m) \\
b_{2}(n)=y_{1}(n)+y_{2}(n)+y_{3}(n)
\end{gathered}
$$

Each of the functions in (2) is expanded polynomially as shown in (3). The coefficients $h_{k}(p, m)$ are then extracted via least squares.

$$
f_{m}^{k}(x)=\sum_{p=0}^{P} h_{k}(p . m) x^{p}
$$

This model is nonlinear in $a_{1}$ and linear in $a_{2}$ and so should be optimal when $a_{2}$ is relatively small. Note that this condition can be described in relation to any load. In fact, if the DUT was operating under a fixed load, then the model should only be dependent on $a_{1}$. In this sense, the least squares algorithm is "free to choose" around which load it is best to settle the behavior of the DUT.

\section{B. DC behavior}

Incorporating the DC current consumption into the DUT model can be difficult because the current measurement is associated with very high time-constants. In fact, the measurable current is not the instantaneous current flowing into the device, but the heavily filtered current flowing from the DC power supply [5].

Using finite impulse response (FIR) like topologies for approximating the current behavior would inevitably lead to extremely long model structures with too many coefficients. To avoid this problem, we have used a first order infinite impulse response (IIR) filter, which allows a very long time-constant using only one coefficient. Using a first order IIR filter also avoids instability problems in the filter solution.

The input to the IIR filter should be the instantaneous current of the device. Even though this current is not known, if a quasi-class B operation is assumed, then the instantaneous DC current, $I_{0}(n)$, is related to the RF current, $I_{1}(n)$, by (4), where $I_{q}$ is the quiescent current and $K$ is a linear gain. In fact, in a textbook class B behavior, the quiescent current would be 0 and the relationship between the currents would be $4 / \pi$.

$$
I_{0}(n) \cong I_{q}+K\left|I_{1}(n)\right|
$$

Furthermore, the RF current of the device can be determined from the incident and reflected waves at the output port, if the matching network is known. This is true because the matching network is a linear network, which means that the waves at the intrinsic transistor plane are a linear combination of the measured waves, and the current is then a linear combination of these intrinsic waves. Even though the matching network is not known, the RF current of the device is 


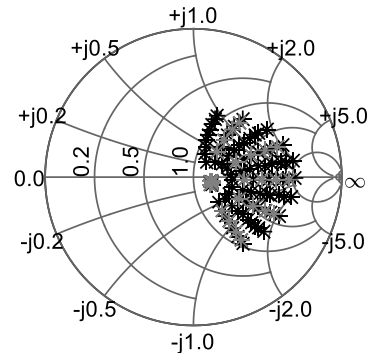

Fig. 3: Measured loads (loads used for extraction in grey).

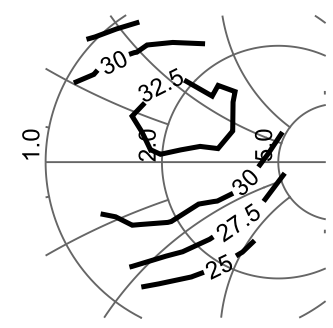

Fig. 5: Efficiency contours (\%) on the measured region.

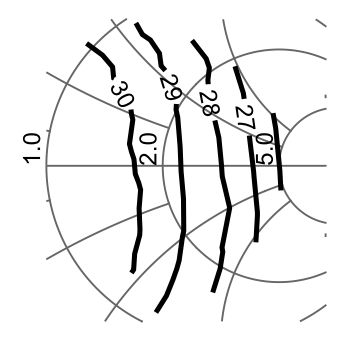

Fig. 4: Average output power contours (in $\mathrm{dBm}$ ).

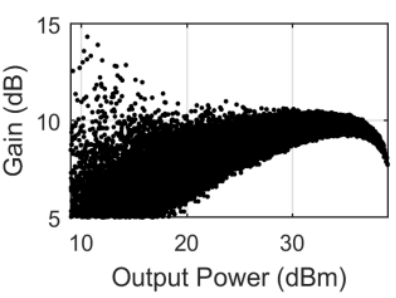

Fig. 6: Gain vs. Output Power for the measured PA. given by equation (5)

$$
I_{1}(n)=C_{1} a_{2}(n)+C_{2} b_{2}(n)
$$

where $C_{1}$ and $C_{2}$ are two model parameters to be extracted. Generally speaking, these coefficients should vary over frequency, in this paper we have initially assumed that the matching network is sufficiently flat over the signal bandwidth that a static approximation is valid.

Since the output wave, $b_{2}$, is known from the RF behavior model, the current model can be compiled from the previous equations as shown in (6), where the IIR filter was converted to sampled time using the bilinear transform.

$$
\begin{gathered}
I_{0}(n)=I_{q}+\left|C^{\prime}{ }_{1} a_{2}(n)+C^{\prime}{ }_{2} b_{2}(n)\right| \\
(1-2 \tau) I_{D C}(n-1)+(1+2 \tau) I_{D C}(n)=I_{0}(n-1)+I_{0}(n)
\end{gathered}
$$

To extract this model, nonlinear optimization is required since the $C_{1}^{\prime}$ and $C^{\prime}{ }_{2}$ coefficients are within a nonlinear function. Note that, in this model, $I_{q}$ and $\tau$ are real, while $C^{\prime}{ }_{1}$ and $C_{2}^{\prime}$ are complex.

\section{RESULTS}

To test the proposed modeling approach we measured a $6 \mathrm{~W}$ Gallium Nitride (GaN) PA (CGH40006 testboard) at $2.6 \mathrm{GHz}$ using orthogonal frequency-division multiplexing (OFDM) signals with specifications similar to a wireless local area network (WLAN) signal, with $16.2 \mathrm{MHz}$ of bandwidth and 10 $\mathrm{dB}$ peak-to-average power ratio (PAPR). The device is measured for varying amplitude and phase of the output incident wave. For each test, the power of the input incident wave, $a_{1}$, is adjusted to maintain the PAPR of the output wave, $b_{2}$, so that the device is operating at approximately the same com-
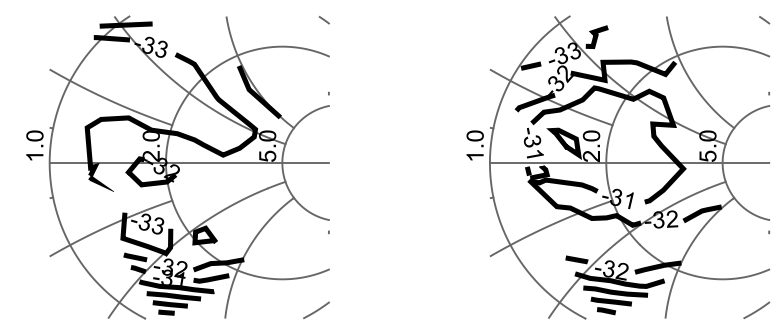

Fig. 7: NMSE between the measured and modelled output wave, for the extraction (on the left) and measured (on the right) signals.
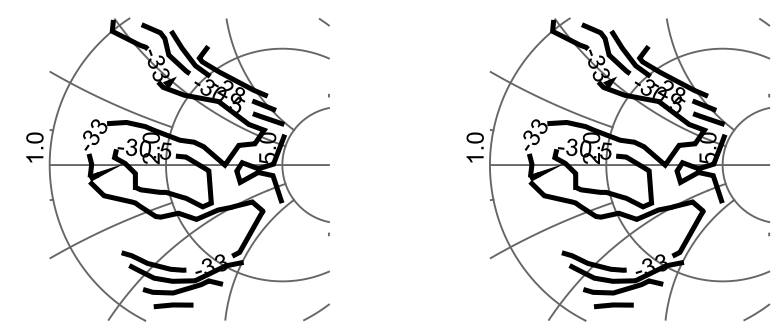

Fig. 8: NMSE between the measured and modelled DC current, for the extraction (on the left) and measured (on the right) signals.

pression level in all measurements. For cross validation, the measurements are repeated for a different WLAN-like signal, where the peak power is adjusted to be the same as the initial measurements.

The phase of the output incident wave, $a_{2}$, was rotated between $[-90,90]$ degrees and the power was swept between $[-30,1] \mathrm{dB}$ with respect to the input incident wave. The powers and angle grids were each divided into 10 points, which resulted in the equivalent synthetized output loads shown in Fig. 3, the average powers of Fig. 4 and the measured efficiencies of Fig. 5. Figure 6 exemplifies the gain profile of the DUT for this particular excitation.

As seen in Figs. 4, 5 and 6, the explored region includes the maximum efficiency region, the DUT is capable of providing about $1 \mathrm{~W}$ of average power with $9 \mathrm{~dB}$ PAPR and a maximum power of about $8 \mathrm{~W}$. The measured maximum efficiency of the $\mathrm{PA}$ is about $33.5 \%$ for this signal.

To test the described models, these were extracted using one quarter of the tested load states (skipping in power and phase) and were validated with both the extraction and validation signals in all the measured loads. The extraction loads are shown in gray in Fig. 3. The RF model parameters in this test were set to 9 for the nonlinear order and 2 for the memory depth. A typical least squares solver was employed to find the optimal solution for the RF model. To extract the current model, the lsqnonlin function from MATLAB was used. This function applies an iterative approach to find an optimal solution. Several solutions are tested before convergence. For each test, the DC current is computed and compared to the measurements for the chosen load conditions.

The normalized mean squared error (NMSE) contours, obtained for both cases, are shown in Fig. 7, for the RF signal, 


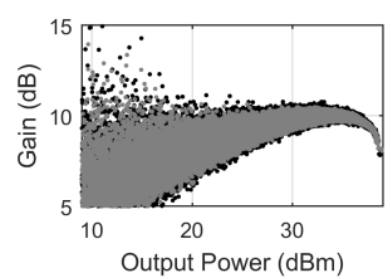

(1)

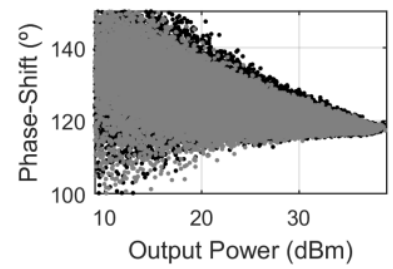

(1)

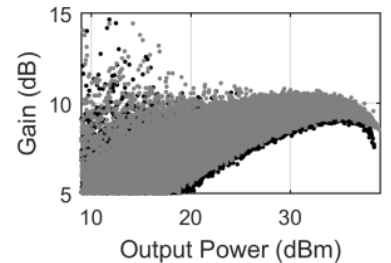

(2)

(a)

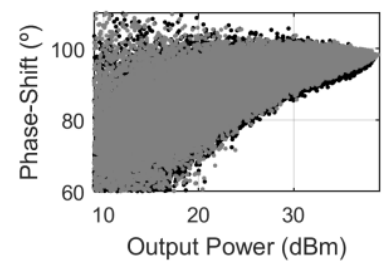

(2) (b)

Measured

Modeled

Fig. 9: AM/AM (a) and AM/PM (b) for the best (1) and worst (2) NMSE.

and in Fig. 8, for the DC current. From the figures, the models achieve an NMSE of $-30 \mathrm{~dB}$ or better in a wide range of loads. Generally speaking, the edge loads show a lower NMSE than the center loads.

For better observation, Fig. 9 shows the measured and modeled AM/AM and AM/PM of the PA for the lowest and highest NMSE. Furthermore, Fig. 10 shows the measured and modeled currents also for the highest and lowest errors. As expected from Fig. 4 and 5, the average output power and average current vary significantly for the different load conditions.

\section{CONCLUSIONS}

One known problem of MIMO systems is the use of non-isolated antenna arrays, which couple the various PAs feeding each antenna element. To simulate these structures at the envelope level, we developed and validated a modeling approach that enables the prediction of both the output envelope and the DC behaviors, allowing efficient system level simulation with linearity, efficiency and output power predictions.

\section{ACKNOWLEDGEMENT}

The authors would like to thank Ampleon, namely Dr. Sérgio Pires, for interesting technical discussions regarding some of the observations dealt within this work. This work is funded

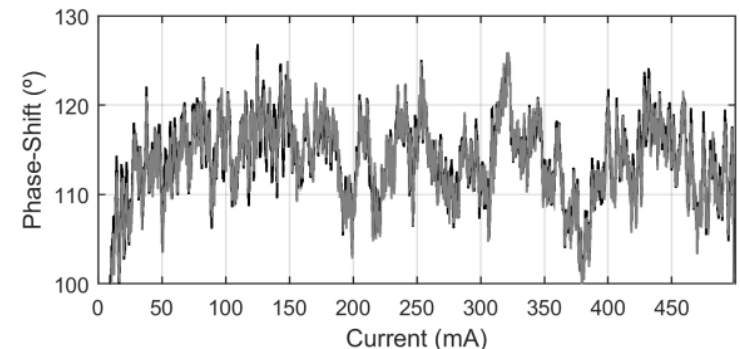

(a)

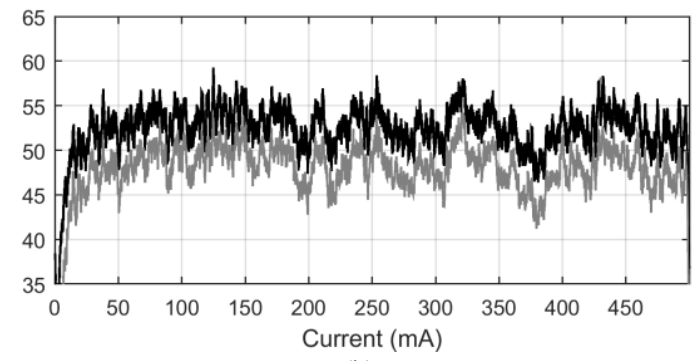

(b)

Measured

Modeled

Fig. 10: Modeled and measured DC current for the best (a) and worst (b) NMSE.

by National Portuguese Funds through FCT - Fundação para a Ciência e a Tecnologia under the project UID/EEA/50008/2013 and PTDC/EEI-TEL/7049/2014, and by the $\mathrm{PhD}$ grant given to the first author (Ref. SFRH/BD/90103/2012).

\section{REFERENCES}

[1] "5G radio access," Ericsson White Paper http://www.ericsson.com/res/docs/whitepapers/wp-5g.pdf, 2013.

[2] F. Rusek et al., "Scaling Up MIMO: Opportunities and Challenges with Very Large Arrays," in IEEE Signal Processing Magazine, vol. 30, no. 1, pp. 40-60, Jan. 2013.

[3] H. Zargar, A. Banai and J. C. Pedro, "A New Double Input-Double Output Complex Envelope Amplifier Behavioral Model Taking Into Account Source and Load Mismatch Effects," in IEEE Transactions on Microwave Theory and Techniques, vol. 63, no. 2, pp. 766-774, Feb. 2015.

[4] C. Fager, X. Bland, K. Hausmair, J. Chani Cahuana and T. Eriksson, "Prediction of smart antenna transmitter characteristics using a new behavioral modeling approach," 2014 IEEE MTT-S International Microwave Symposium (IMS2014), Tampa, FL, 2014, pp. 1-4.

[5] J. C. Pedro, P. M. Cabral, T. R. Cunha and P. M. Lavrador, "A Multiple Time-Scale Power Amplifier Behavioral Model for Linearity and Efficiency Calculations," in IEEE Transactions on Microwave Theory and Techniques, vol. 61, no. 1, pp. 606-615, Jan. 2013. 